


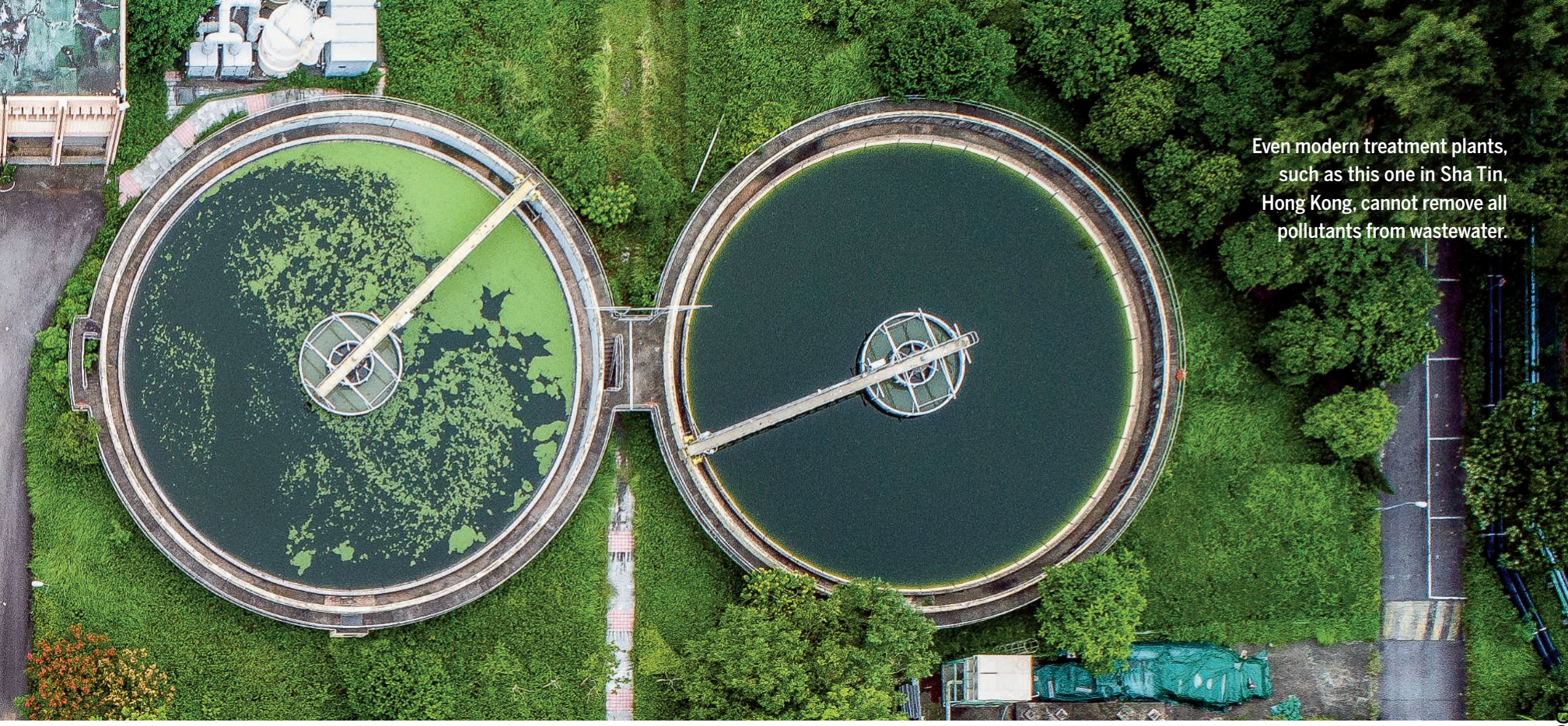

example, is recovered and reused in dyeing, as are sizing chemicals and unused indigo from denim dyeing.

Separation at the source will be much more challenging for municipal wastewater, where it will require a focus on the chemicals used in products. This may involve selection of biodegradable compounds, use of fewer constituents, and use of smaller amounts of chemicals overall.

For example, organic silicon compounds were long seen as indispensable in shampoos. However, they do not fully degrade in the environment, and their degradation products are ubiquitous. Following increasing consumer awareness, producers have begun to promote silicon-free alternatives, saving money in the process because they require fewer ingredients. Similarly, replacing plastic microbeads with fully and readily mineralizable cellulose microbeads in cosmetics would avoid the release of long-lived microplastics into the environment. Given the numerous ingredients of cosmetic and other products, there should be many such opportunities for reducing the environmental burden of synthetic chemicals.

Recent developments in green (9) and sustainable chemistry (10), including pharmaceutical chemistry, also offer approaches for input reduction or prevention. One promising approach is to understand the reasons for use of a chemical substance, determine which functionality it offers, and explore nonchemical alternatives. For example, different construction or design of a building may eliminate the need for fungicides, if wooden parts are only used where water has no access. Similarly, different consumer behavior or alternative business and serviceoriented models could reduce the amount or nature of chemicals used $(11,12)$.

\section{BENIGN BY DESIGN}

Even if input can be reduced, it is obvious that chemicals will continue to be needed, albeit perhaps in smaller amounts. Ideally, any such chemicals that remain in wastewaters should be designed such that they are rapidly and completely mineralized (degraded to carbon dioxide, water, and mineral species) in effluent treatment or even in surface waters. For example, linear alkyl sulfonate was developed for quick and complete mineralization over 50 years ago as a substitute for the persistent synthetic detergent tetrapropylene sulfonate. It is thus feasible to intentionally design chemicals in such a way that treatment or processes in the environment would lead to fast and complete mineralization ("benign by design").

Accordingly, to be environmentally benign, the chemicals and products of the future must be assessed to meet this requirement at the very beginning of their life cycle, taking into account the end of their life even before their synthesis. There is limited chemical freedom to vary the core parts of molecules that are essential for their function. However, other parts can be varied much more to foster quick and complete mineralization in conventional wastewater treatments or aquatic environments (13). Encouraging examples are available even for widely used pharmaceuticals, such as $\beta$-blockers (14), and chemicals, such as ionic liquids (15).

\section{GOING BEYOND INDIVIDUAL APPLICATIONS}

Although examples of more benign chemicals exist, going beyond these individual applications requires improved knowledge and management of the substance, material, and product flows in the global economy. Knowledge of the local, regional, national, and global variations and dynamics of these flows would help to identify opportunities and levers for reducing their chemical complexity. Better knowledge of products, their targeted design, and an enhanced understanding of the function they offer are key for achieving this goal.

The current trend is, however, in the opposite direction, with ever more complex products with diverse composition entering the market and hence the environment. It is thus crucial that incentives and regulation are in place to steer product and chemical development in a more sustainable direction. To achieve such a change in direction, external costs such as wastewater treatment or environmental cleanup costs must be taken into account in product price. For instance, a slight modification in the production process to increase revenue may result in substantial external costs for wastewater and drinking water management, and vice versa.

Regulations and incentives are needed to make this happen. For example, introduction of the European chemicals regulation REACH reduced the number of chemicals on the European market as manufacturers balanced registration costs against possible revenues. In the future, fast-track registration or prolonged patent lifetime could help to incentivize the development of new compounds designed for fast and complete mineralization in the environment. Chemicals that are readily mineralized in the environment need not be as extensively tested for effects in the environment, reducing costs and saving time.

REGULATION FOR INDIVIDUAL CHEMICALS

In an ever more complex and interdependent world, the precautionary principle in general, as well as with respect to chemical input prevention at the source, becomes more important. What's going on at the beginning of the pipe determines what leaves the end of the 
pipe, not the other way around. The amendment of water regulations presents an ideal opportunity to promote source control and drive innovation. Yet sewerage charges, if in place at all, are typically applied to cover the costs for (re)building sewers and operating sewage treatment plants.

In some countries, for example in Germany, there is an additional fee for the release of water that does not meet certain quality criteria. These fees are measured by sum parameters such as chemical oxygen demand and nitrogen and phosphorus content. There are also threshold concentrations for organic halogens and metals such as mercury, cadmium, chromium, lead, and copper, based on their toxicity to fish eggs. This approach has been very effective for input prevention, resulting, for example, in reduced use or recovery of halogenated solvents. It could be extended to persistent organic compounds in general, including specific toxicity such as mutagenicity, genotoxicity, and endocrine-disrupting potential. Bans for long-lived pollutants will help to reduce concentrations of pollutants that contribute little to the utility of products.

However, given the ever-increasing list of chemicals that are introduced into the aquatic environment, attempts to assess harm and introduce thresholds will tend to lag new introductions. A preventive approach is therefore also needed. For example, giving companies relief from effluent charges if they use compounds from a list proven to be of low toxicity and readily mineralized-such as the abovementioned cellulose microbeadscould provide strong incentives for creating more sustainable products.

\section{REFERENCES \\ 1. E. S. Bernhardt, E. J. Rosi, M. O. Gessner, Front. Ecol. Environ. 15, 84 (2017). \\ 2. R.P.Schwarzenbachetal.,Science 313,1072 (2006) \\ A. Christou etal., Water Res. 109,24(2017). \\ O. Paltiel et al., Environ. Sci. Technol. 50, 4476 (2016) \\ 5. C. K.Schmidt, H.-J.Brauch, Environ. Sci. Technol. 42,6340 (2008). \\ 6. D. Haniganetal. Environ. Sci. Technol.Lett. 2.151(2015). \\ 7. F.Murphy etal.,Environ. Sci. Technol.50,5800 (2016). \\ 8. J.Alexander, G. Knopp, A. Dötsch, A. Wieland, T.Schwartz, Sci. Total Environ. 559, 103 (2016) \\ 9. J.A. Linthorst, Found Chem 12,55(2010) \\ 10. K. Kümmerer,Angew. Chem. Int. Ed. 56,16420 (2017). \\ 11. United Nations Industrial Development Organization, Global Promotion and Implementation of Chemical Leasing Business Models in Industry (UNIDO, Vienna, 2016). \\ 12. C.G. Daughton, Sci. Total Environ. 493,392 (2014). \\ 13. R.S. Boethling, E. Sommer, D. DiFiore, Chem. Rev. 107, 2207 (2007). \\ 14. T. Rastogi, C. Leder, K. Kümmerer, Environ. Sci. Technol. 49 11756 (2015). \\ 15. A. Haißetal., Green Chem. 18, 4361(2016).}

SUPPLEMENTARY MATERIALS

www.sciencemag.org/content/361/6399/222/suppl/DC1

10.1126/science.aau2405

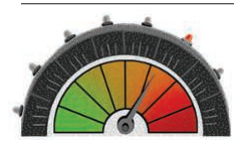

TOMORROW'S EARTH

Read more articles online

at scim.ag/TomorrowsEarth

\section{CHEMISTRY}

\section{Regulate to reduce chemical mixture risk}

\section{Regulatory systems must better provide for risks from exposure to multiple chemicals}

\section{By Andreas Kortenkamp ${ }^{1}$ and Michael Faust ${ }^{2}$}

I umans and wildlife are continuously exposed to multiple chemicals from different sources and via different routes, both simultaneously and in sequence. Scientific evidence for heightened toxicity from such mixtures is mounting, yet regulation is lagging behind. Ensuring appropriate regulation of chemical mixture risks will require stronger legal stimuli as well as close integration of different parts of the regulatory systems in order to meet the data and testing requirements for mixture risk assessment.

Until about a decade ago, toxicologists, risk assessors, and regulators regarded risks from chemical mixtures as negligible, as long as exposures to all single chemicals in the cocktail were below the levels judged to be safe for each chemical alone $(1,2)$. However, an increasing body of scientific evidence has challenged this notion, showing that a neglect of mixture effects can cause chemical risks to be underestimated (see the figure). International bodies such as the World Health Organization now acknowledge the need for considering mixtures in chemical risk assessment and regulation (3). This would align toxicological risk assessment with the clinical sciences and their long tradition of investigating drugdrug interactions. Yet, with few exceptions, regulatory systems around the world still focus overwhelmingly on single-chemical assessments, and the translation of scientific evidence about mixture effects into better regulation is extremely slow.

\section{THE SCIENTIFIC BASIS}

The most widely used concept to determine the common toxic effect of combinations of chemicals is dose addition (DA) $(3,4)$. DA assumes that one chemical can be replaced by an equal fraction of an equally effective dose of another without diminishing the overall combined effect. This may, for example, be the case for combinations of chemicals that exert their toxicity through similar mechanisms, such as by binding to the same receptor.
In one of the earliest predictive mixture studies, DA provided good approximations of the joint effects of mixtures of 50 aquatic toxicants in fish (5). This seminal paper created the conceptual basis for numerous laboratory studies with microbes, mammalian cells, rodents, and isolated human tissues. In these studies, DA proved to be an excellent tool for anticipating experimentally observed combination effects of up to 80 chemicals, including pesticides, industrial chemicals, food contaminants, cosmetics ingredients, and pharmaceuticals $(6,7)$.

The principles of DA imply that every mixture component contributes to the combination effect in proportion to its dose and individual potency, even when each component is present at levels below its individual effect threshold. This idea has been tested in several experimental studies. The results show that mixture effects occurred when each chemical was present at or below experimental NOAELs (no observed adverse effect levels) for single substances $(8)$. NOAELs are used to derive regulatory limit values through division by an assessment factor, typically 100 . Examples are Environmental Quality Standards (EQS) set under the European Water Framework Directive.

The suitability of such EQS for protecting against mixture effects has been tested. Combinations of 14 or 19 pollutants at EQS levels produced substantial toxic effects in microalgae, daphnids, and fish and frog embryos (9), at concentrations 100-fold or more below their individual NOAELs. A mixture of 15 chemicals at the concentrations found in human amniotic fluid altered thyroid hormone signaling and early brain development in Xenopus tadpoles (10).

Clearly, single-chemical risk assessments cannot capture such phenomena. Mixture risk assessment is needed for better protection of humans and the environment. Scientifically justifiable tools are available and ready for use in risk-assessment practice.

IInstitute of Environment, Health and Societies, Brunel University London, Uxbridge UB8 3PH, UK. ${ }^{2}$ Faust \& Backhaus Environmental Consulting, Fahrenheitstrasse, Bremen, Germany. Email: andreas.kortenkamp@brunel.ac.uk 


\section{Science}

\section{A path to clean water}

Klaus Kümmerer, Dionysios D. Dionysiou, Oliver Olsson and Despo Fatta-Kassinos

Science 361 (6399), 222-224.

DOI: $10.1126 /$ science.aau2405

ARTICLE TOOLS

SUPPLEMENTARY MATERIALS

RELATED

CONTENT

REFERENCES

PERMISSIONS http://science.sciencemag.org/content/361/6399/222

http://science.sciencemag.org/content/suppl/2018/07/18/361.6399.222.DC1

http://science.sciencemag.org/content/sci/361/6399/224.full

http://science.sciencemag.org/content/sci/361/6399/eaam5324.full file:/content

This article cites 14 articles, 1 of which you can access for free http://science.sciencemag.org/content/361/6399/222\#BIBL

http://www.sciencemag.org/help/reprints-and-permissions

Use of this article is subject to the Terms of Service

Science (print ISSN 0036-8075; online ISSN 1095-9203) is published by the American Association for the Advancement of Science, 1200 New York Avenue NW, Washington, DC 20005. The title Science is a registered trademark of AAAS.

Copyright $@$ 2018, American Association for the Advancement of Science 